\title{
Simulation of the Double-closed Loop Three-phase Asynchronous Variable Voltage Control System
}

\author{
Shuhua Jiang \\ School of Electronic Information Engineering, Changchun University of Science \& Technology \\ Changchun 130022, China \\ E-mail: jiangeye@126.com
}

Received: July 6, 2011

Accepted: August 10, 2011

doi:10.5539/mas.v5n5p214

\begin{abstract}
When controlling the voltage and speed of the asynchronous motor, to expand the speed control range and reduce the speed fluctuation, the speed control system adopts two feedback control loops, i.e. the speed feedback control loop and the current feedback control loop. The principle and components of the double-closed loop three-phase asynchronous variable voltage control system are studied, and the simulation model and the test result based on MATLAB/Simulink are provided in this article through the system modeling and the parameter setup of the main circuit and the control circuit.
\end{abstract}

Keywords: Double-closed loop, Asynchronous motor, AC voltage regulator

\section{Variable voltage control principle}

In variable voltage control of asynchronous motor, because the synchronous speed is invariant and the mechanical character is rigid, the speed adjustable range is limited for common asynchronous motor, without practical value, but for moment motor or coiling asynchronous motor, the mechanical character will become soft if the proper resistance is bunched into the motors, and the speed adjustable range will be expanded, and even the speed fluctuation becomes very obvious when the loads or the voltages of the grid fluctuate, so the double-closed loop variable voltage control system is often adopted.

The functional block diagram of the system is seen in Figure 1.

The system adopts two feedback control loops including the speed feedback control loop and the current feedback control loop. The starting time and the braking time decide the production efficiency of the production machine to a large extent. To reduce the start, braking, and rollback time of the motor, the revolving speed negative feedback of the proportional integral controller is adopted in the system. When running stably, the current loop has good anti-jamming function for the fluctuation of the grid, but in the starting process, the current loop could only limit the maximum current, and the constant-current characteristics of the best starting will not occur, and the constant torque starting will not occur too.

The variable voltage control structure of asynchronous motor is simple, and the static voltage error is small when the double-closed loop system is adopted, and the rollback, the inversed joint, and the dynamic braking, are easy to be realized. $\mathrm{Bu}$ in the load of constant torque, the system could not run in lower speed, because in lower speed, the slip power $\mathrm{P}_{\mathrm{S}}=\mathrm{SP}_{\mathrm{M}}$ will completely be consumed in the rotor resistance, and the rotor will become too hot.

\section{System simulation}

The principal circuit of the variable voltage control structure of double-closed loop 3-phase asynchronous motor is composed by the 3-phase SCR AC voltage regulator and the 3-phase coiling asynchronous motor. The control part is composed by "current regulator", "speed change", "trigger circuit", and "proper bridge amplifier". The electric principle diagram of the AC variable voltage system is seen in Figure 1. The simulation model of the AC variable voltage system is seen in Figure 2.

\section{Modeling and parameter setup of main circuit}

From Figure 2, the principal circuit is composed by 3-phase AC voltage regulator, AC asynchronous motor, and the motor signal distributor. 


\subsection{Modeling and parameter setup of three-phase alternating-current supply}

The mutual phase difference of 3-phase power supply is $120^{\circ}$, and the setup AC maximum value phase voltage is $100 \mathrm{~V}$, and the frequency is $60 \mathrm{~Hz}$.

\subsection{Modeling and parameter setup of three-phase AC voltage regulator of SCR (silicon controller rectifier)}

The SCR three-phase AC voltage regulator is composed by three pairs of antiparallel SCR components, and each SCR component is controlled by the mode of "phase control", and the current is converted naturally according to the grid current. Figure 3 is the simulation model of the SCR three-phase AC voltage regulator, and in Figure 3, single SCR component is linked as the simulation model according to the connection requirement of three-phase AC voltage regulation. Figure 4 is the parameter setup of the SCR components in the three-phase AC voltage regulator.

\subsection{Modeling and parameter setup of the AC asynchronous motor and the motor test signal distributor}

In the tool box of "PowerSystem", there is a motor module library, which contains the DC motor module, the asynchronous motor module (PU unit and SI unit), the synchronous motor module, and other motor modules, and the asynchronous motor module in SI unit is adopted in this article.

The state equation to describe the module performance of asynchronous motor includes the electric part and the mechanical part. The electric part includes 5 state equations, and the mechanical part includes 2 state equations. This module has 4 input terminals and 4 output terminals. The former 3 input terminals $(A, B, C)$ in the module are the stator voltage input of the motor, and the fourth output terminal is often linked with the load, and it is the mechanical load on the motor, and this terminal can directly link the signal of SIMULINK. The former 3 output terminals $(\mathrm{a}, \mathrm{b}, \mathrm{c})$ of the module are the rotor voltage outputs linking together by the short connected mode, or linking with other additional circuits, and when the asynchronous motor is the squirrel-cage motor, the symbols of the motor module will not be displayed by output terminals ( $a, b, c)$. The fourth output terminal is $\mathrm{m}$ terminal, and it returns a series of internal signals of the motor (including 15 paths and 21 parameters), and provides the input $\mathrm{m}$ terminals for the motor test signal distributor module. The output signals can be selected according to the actual test requirements, through setting up the dialog box of the motor test signal distributor module, and the physical quantity can be selected freely. The parameter selection of the dialog frame setup of the motor test signal distributor is seen in Figure 4.

The parameters of the asynchronous motor can be inputted through the parameter dialog frame of the motor module, including following parameters.

Rotor type list box: Would type and Squirrel-cage type. The latter is selected in this article.

Reference frame list box: Stationary frame, Rotor coordinate system, Synchronous rotating coordinate system which is selected in this article.

Rated parameters: Rated power $\mathrm{P}_{\mathrm{n}}(\mathrm{kW})$, Line voltage $\mathrm{V}_{\mathrm{n}}(\mathrm{V})$, Frequency $f(\mathrm{~Hz})$.

Stator resistance $\mathrm{R}_{\mathrm{s}}(\Omega)$, Leakage inductance $\mathrm{L}_{\mathrm{ls}}(\mathrm{Hz})$.

Rotor resistance $\mathrm{R}_{\mathrm{r}}(\Omega)$, Leakage inductance $\mathrm{L}_{\mathrm{lr}}(\mathrm{Hz})$.

Mutual inductance $\mathrm{L}_{\mathrm{m}}(\mathrm{Hz})$.

Rotary inertia $\mathrm{J}\left(\mathrm{kg} \cdot \mathrm{m}^{2}\right)$, Polar logarithm P.

The parameter setup of the dialog frame of asynchronous motor is seen in Figure 5.

\section{Modeling and parameter setup of control circuit}

The control circuit of the $\mathrm{AC}$ variable voltage control system includes the given part, the speed regulator, the amplitude limiter, and the speed feedback part.

\subsection{Modeling and parameter setup of the given signal module}

In the input \& output module, the module of "Constant" is selected, and the module parameter is set by 100rad/s. When controlling the speed in the practice, the allowed change range of given signals can be confirmed according to the simulation practice.

\subsection{Modeling and parameter setup of amplitude limiter module}

From the simulation experiment of the $\mathrm{U}_{\mathrm{ct}}$ parameter change range, when $U_{c t}$ changes in $50^{\circ} \sim 180^{\circ}$, the 
synchronic pulse trigger could work normally. And when $\mathrm{U}_{\mathrm{ct}}$ is $50^{\circ}$, the corresponding rectifier bridge output voltage is maximum, and the corresponding output voltage of $180^{\circ}$ is minimum, and they have the monotonously descending function relationship. The parameter setup of amplitude limiter is seen in Figure 6 .

\subsection{Parameter setup of system simulation}

The system simulation model (seen in Figure 2) can be obtained by linking the simulation model of the principal circuit and the control circuit according to the link relationship of the double-closed loop three-phase asynchronous motor variable voltage system electric schematic diagram.

The speed feedback system is set by $30 / \mathrm{pi}$, and in the speed regulator, $X_{\mathrm{p}}=40$, and $\tau_{\mathrm{n}}=300$. The simulation algorithm is ode23tb. The "Start time" of the simulation is 0 , and the "Stop time" is 4.2.

\section{Output of simulation results}

After modeling and parameter setting, the simulation could start. The simulation result is seen in Figure 7. From the simulation result, in stable state, the actual speed of the simulation system could follow the given speed well, but in the transient process, the actual speed of the simulation system has certain errors to follow the stepped signals.

\section{Conclusions}

The variable voltage control structure of double-closed loop three-phase asynchronous motor is discussed and studied in this article, and the speed control system adopts the speed feedback control loop and the current feedback control loop. The control circuit of the speed control system is composed by the current regulator, the speed convertor, and the trigger current. From the simulation result of MATLAB/Simulink, the time speed in the variable voltage control system could follow the given speed very well.

\section{References}

Deng, Jianguo \& Luo, Longfu. (2008). Simulation for Electric Braking of Single-phase Capacitance Motors with Semihex $\sim(\mathrm{TM}) \sim$ connected. Journal of System Simulation. No. 1.

Gao, Rui. (2005). Study on the Energy Conservation Protector of Three-phase Asynchronous Motor. Chengdu: Thesis of Sichuan University.

Wang, Sujiao. (2006). Selection of Capacitance in Three-phase Motor Running as Single-phase Motor. Electric Machines \& Control Application. No. 7.

Zhang, Xiaofeng. (2007). Development and Prospection of the AC Motor Control Technology. Electronic Products China. No. 6.

Zhong, Chao. (2010). Design and Implementation of the Three-phase Asynchronous Motor Direct Torque Control System. Wuhan: Wuhan University of Technology. 


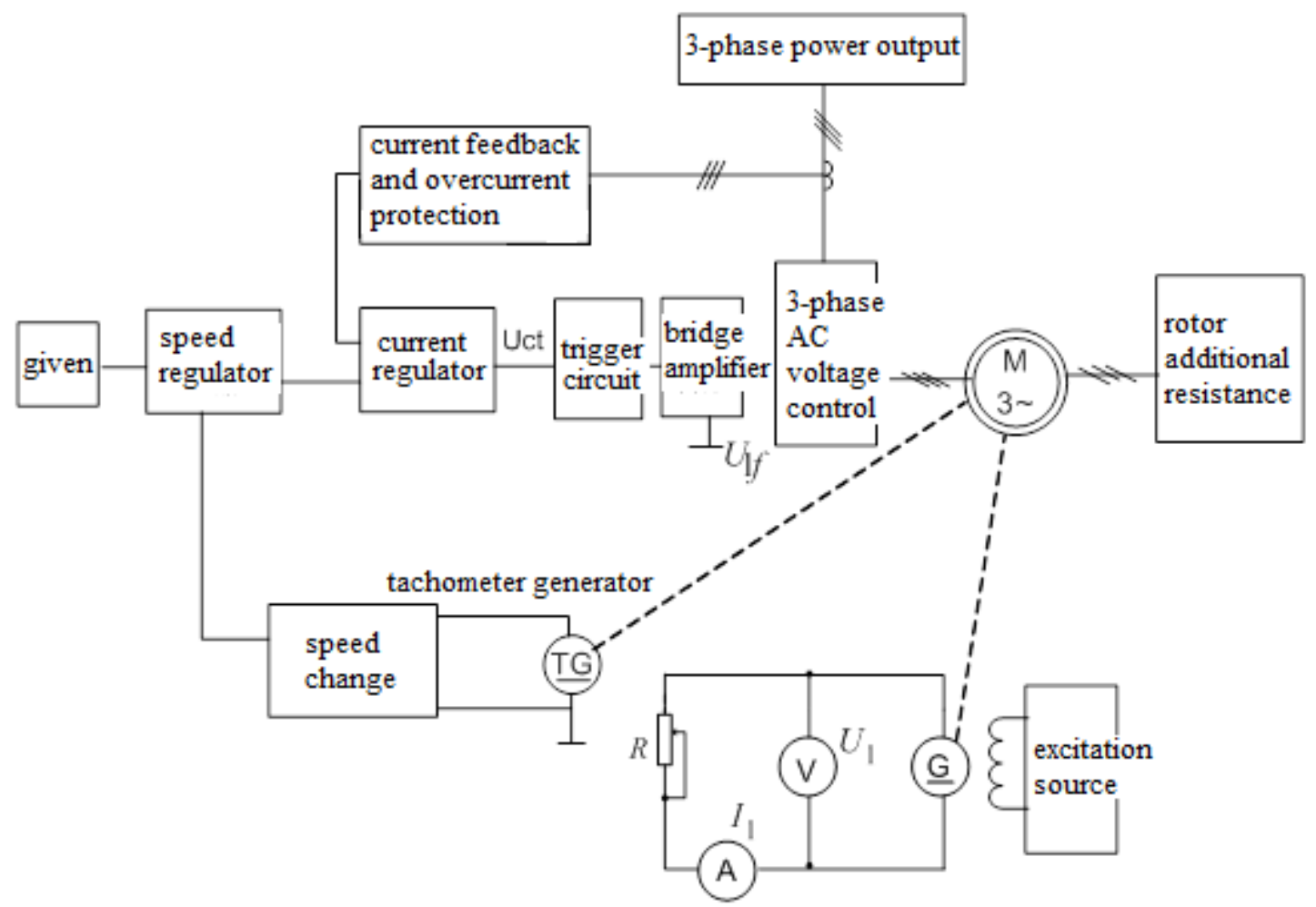

Figure 1. Functional Block Diagram of the Double-closed Three-phase Asynchronous Motor Variable Voltage Control System

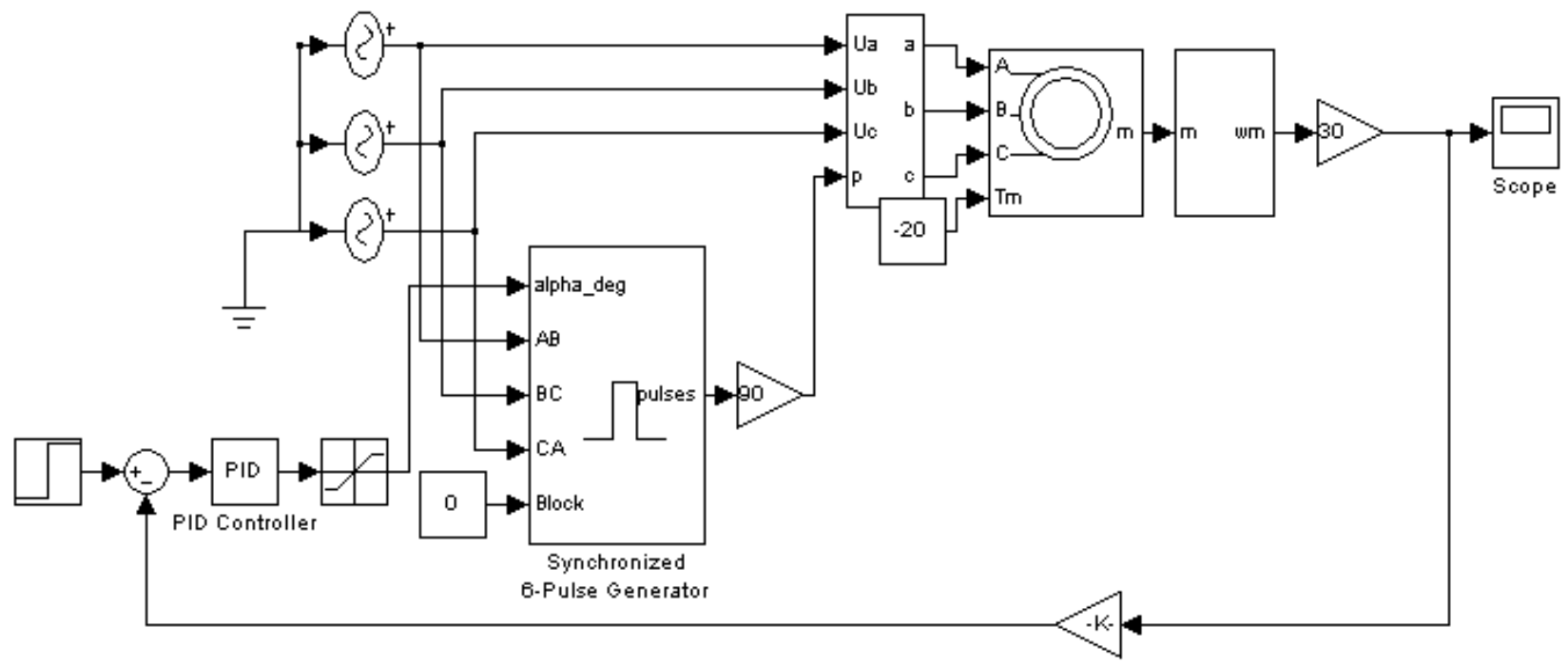

Figure 2. Simulation Model of the AC Variable Voltage Control System 


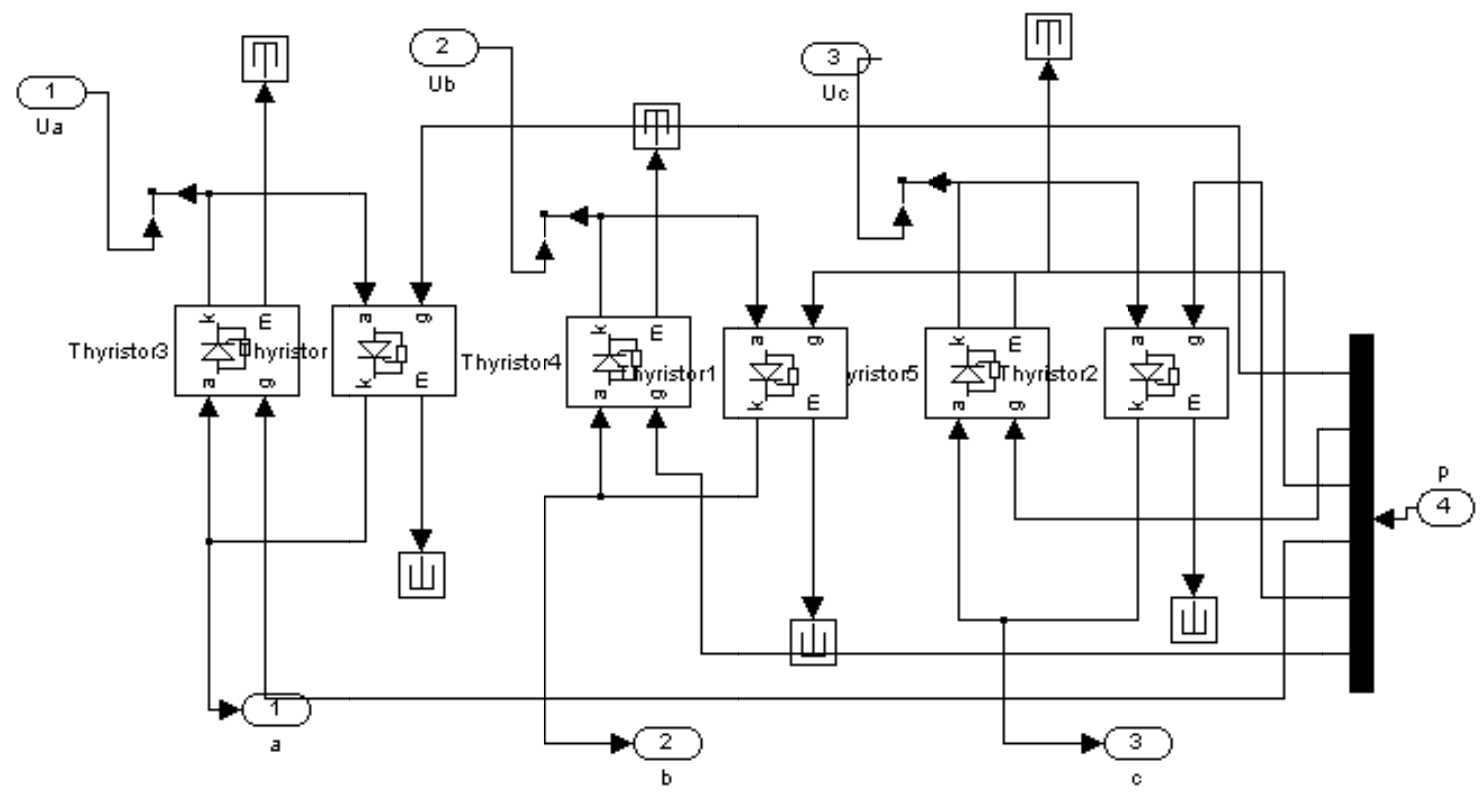

Figure 3. Simulation Model of the Three-phase AC Voltage Regulator of SCR

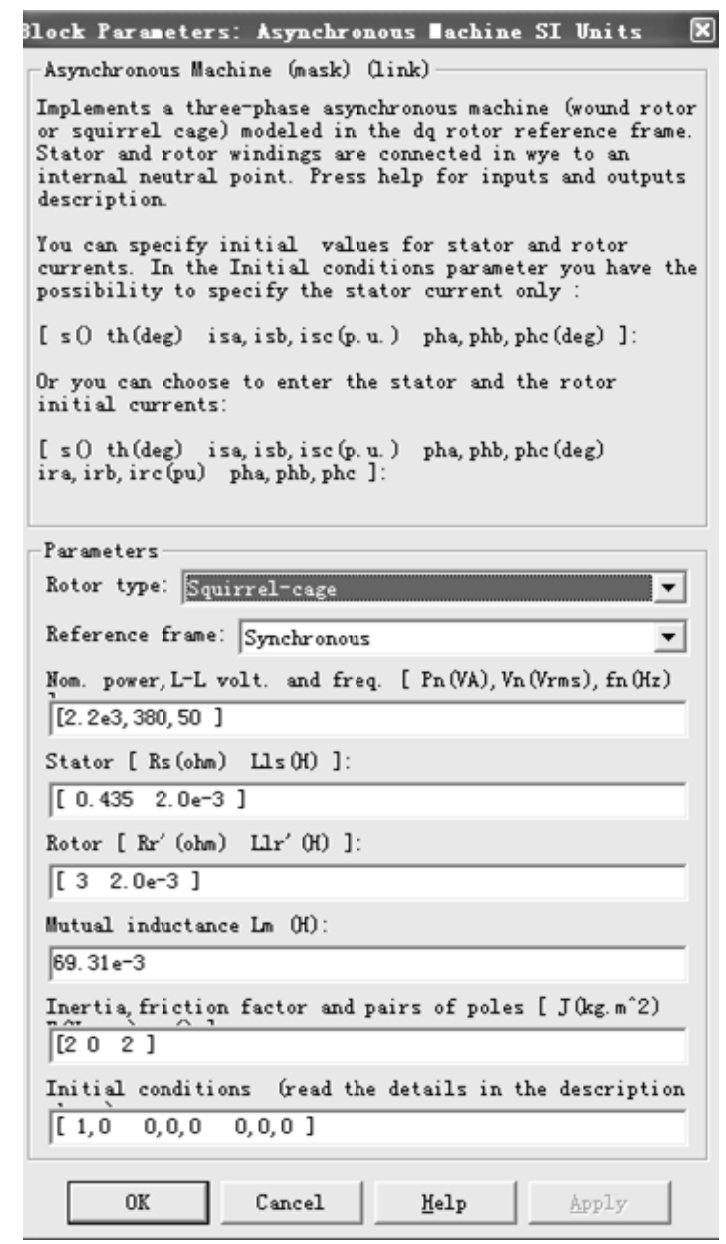

Figure 4. Parameter Setup of the Motor Test Signal Distributer 


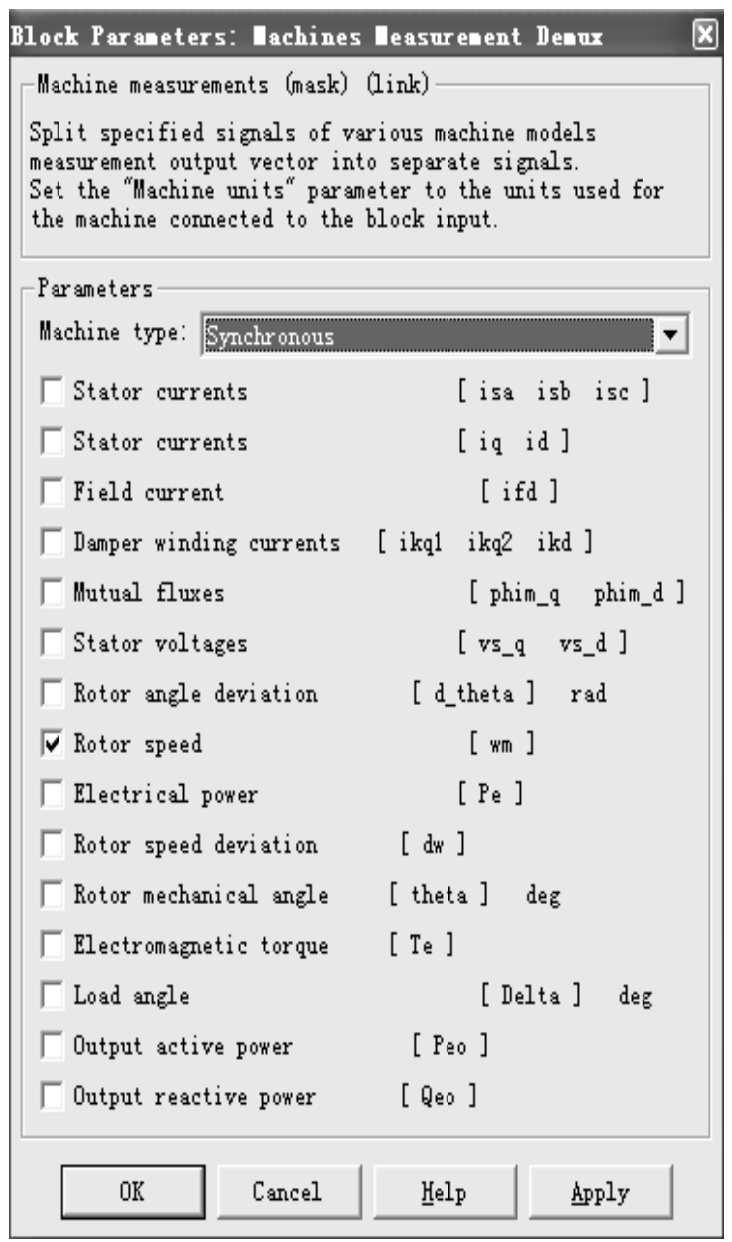

Figure 5. Parameter Setup of Asynchronous Motor

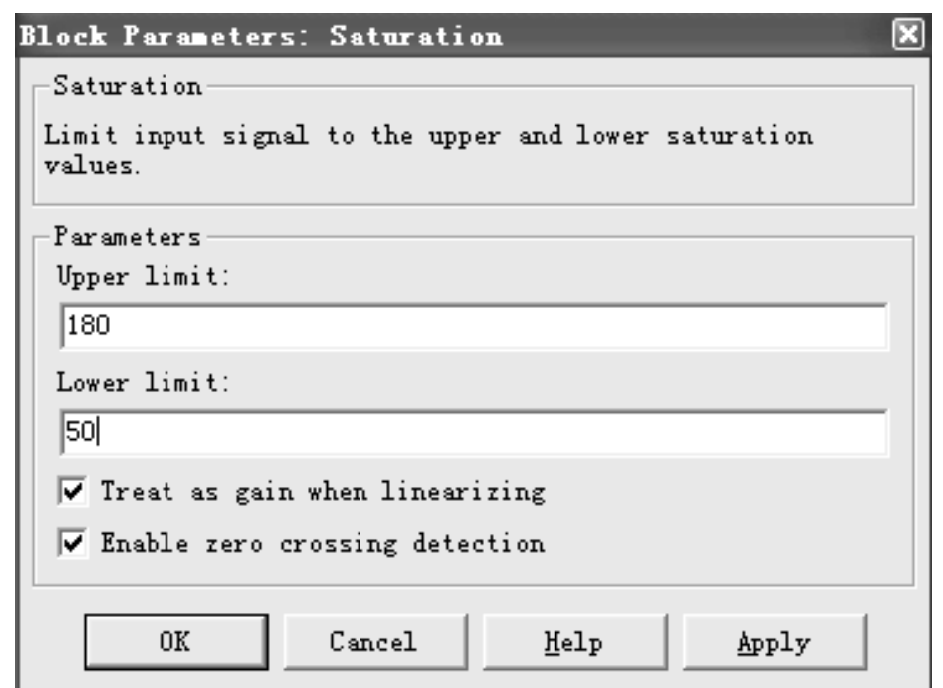

Figure 6. Parameter Setup of Amplitude Limiter 


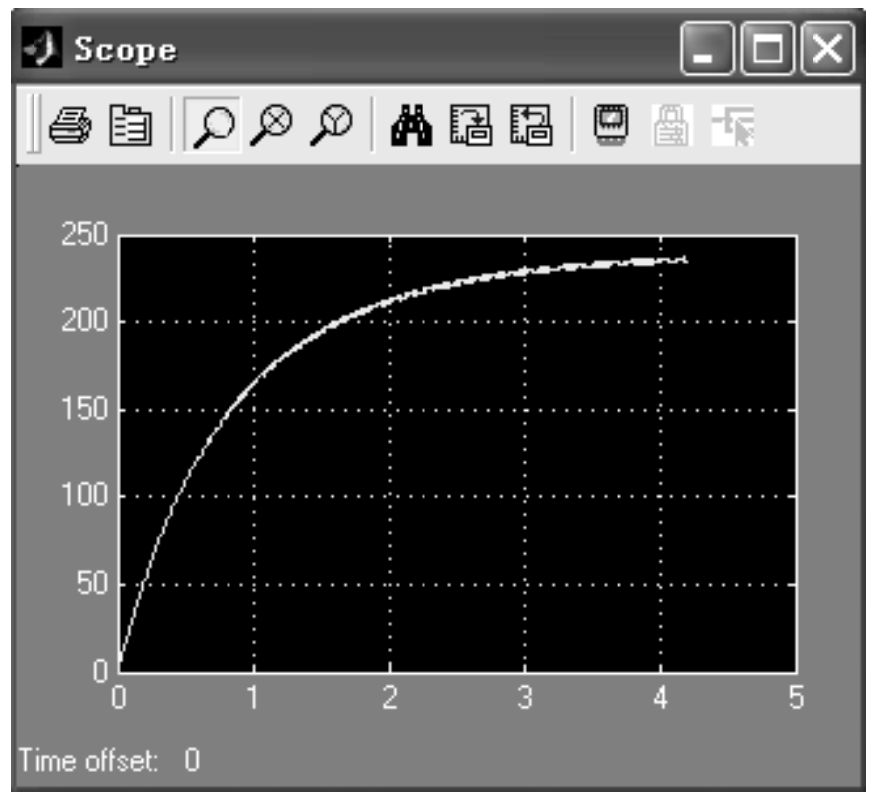

Figure 7. Rotated Speed of the AC Variable Voltage Control System 